
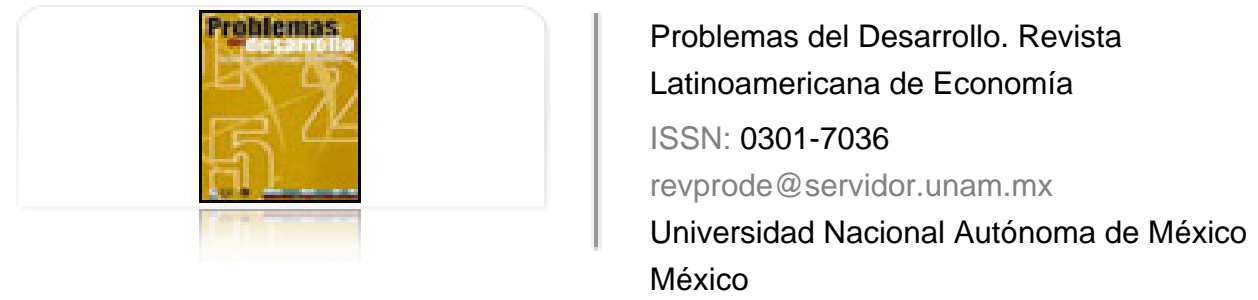

Lira, Adrián

La tecnología de la información y la comunicación: estudio económico Problemas del Desarrollo. Revista Latinoamericana de Economía, vol. 36, núm. 143, octubrediciembre, 2005, pp. 189-212

Universidad Nacional Autónoma de México

Distrito Federal, México

Disponible en: http://www.redalyc.org/articulo.oa?id=11820081009

- Cómo citar el artículo

Número completo

- Más información del artículo

Página de la revista en redalyc.org

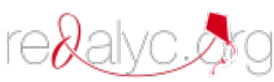

Sistema de Información Científica

Red de Revistas Científicas de América Latina, el Caribe, España y Portugal Proyecto académico sin fines de lucro, desarrollado bajo la iniciativa de acceso abierto 


\section{LA TECNOLOGÍA DE LA INFORMACIÓN Y LA COMUNICACIÓN: ESTUDIO ECONÓMICO}

Adrián Lira*

Fecha de recepción: 4 de abril de 2005. Fecha de aceptación: 6 de octubre de 2005.

\section{Resumen}

La tecnología de la información y comunicación (TIC) está provocando profundos cambios en las esferas económica, política y social en función de sus diversas modalidades de presentación en el mundo, que han incentivado su exploración conceptual en distintos ámbitos de competencia. Los esfuerzos teóricos que tratan el actual fenómeno tecnológico han derivado en un conjunto multifacético de estudios de caso a partir de los cuales se estructura el desenvolvimiento tecnoeconómico de la TIC en las sociedades internacionales. Por ello, este trabajo compila, sintetiza y expone algunos de los más plausibles adelantos teóricos en la temática y los organiza de tal forma que los actuales cambios tecnoeconómicos, nacionales e internacionales, sean comprendidos desde una perspectiva económica evolucionista.

Palabras clave: tecnología de la información y comunicación, desenvolvimiento tecnológico, sociedad de la información, economía digital, Internet.

\section{Abstract}

Information and communication technology (ICT) is leading to profound changes in the economic, political and social spheres as a result of its diverse modes of presentation in the world, giving incentives to its conceptual exploration in various areas of competition. The theoretical efforts dealing with this contemporary technological phenomenon have led to a multifaceted group of case studies from which is structured the techno-economic performance of ICT in international societies. This study, therefore, compiles, synthesizes and expounds on some of the more plausible theoretical advances in the area and organizes them in such a way that the present techno-economic, national and international changes are comprehended from an evolutionary economics perspective.

Key words: information and communication technology; technological performance; information society; digital economy; Internet.

* Profesional ejecutivo de servicios especializados, Subdirección de Estadísticas Informáticas, INEGI. Correo electrónico: Ramiro.Lira@inegi.gob.mx 


\section{Résumé}

La technologie de l'information et de la communication (TIC) provoque de profonds changements dans les sphères économique, politique et sociale en fonction de ses diverses modalités de présentation dans le monde, qui ont encouragé son exploration conceptuelle dans différents environnements de concurrence. Les efforts théoriques qui traitent le phénomène technologique contemporain ont dérivé vers un ensemble multifacettes d'études de cas à partir desquels on structure le déroulement technoéconomique des TIC dans les sociétés internationales. C'est pourquoi ce travail rassemble, synthétise et expose quelques uns des progrès théoriques les plus plausibles dans la thématique et les organise d'une façon telle que les changements technoéconomiques, nationaux et internationaux, soient compris depuis une perspective économique évolutionniste.

Mots-cléfs: technologie de l'information et de la communication, déroulement technologique, société de l'information, économie numérique, Internet.

\section{Resumo}

A tecnología da informação e comunicação (TIC) está causando profundas mudanças nas esferas econômica, política e social em função das suas diversas modalidades de apresentação no mundo, que têm incentivado sua exploração conceitual em distintos âmbitos de competência. Os esforços teóricos que tratam o atual fenómeno tecnológico contemporâneo derivaram num conjunto multifacético de estudos de caso a partir dos quais estrutura-se o desenvolvimento tecno-econômico da TIC nas sociedades internacionais. Por isto, este trabalho colige, sintetiza e expõe alguns dos mais plausíveis abanicos teóricos na temática e organiza-os de tal jeito que as mudanças tecno-econômicas atuais nacionais e internacionais sejam compreendidas desde uma perspetiva econômica evolucionista.

Palavras chave: tecnologia da informação e comunicação, desenvolvimento tecnológico, sociedade da informação, economia digital, Internet.

\section{DeSarrollo}




\section{Introducción}

$\mathrm{E}$

1 fenómeno contemporáneo de la tecnología de la información y las comunicacio-

nes (TIC) produce cambios profundos en las sociedades dentro de las esferas eco-

nómica, política y social de inicios de siglo, con más relevancia en las naciones con mayor desarrollo económico y tecnológico que en aquellas con menor grado. ${ }^{1}$

El actual desenvolvimiento internacional del uso y aplicación económica de la TIC ha llevado a plantear una serie de conceptos en los resultados sociales del uso de esta tecnología. Muchos términos han surgido, algunos con matices mercadológicos, otros a manera de discursos rectores y otros más como planteamientos académicos y filosóficos. No obstante, la realidad muestra la falta de una línea teórica pura en el análisis económico del fenómeno, lo cual se debe a la complejidad en la abstracción del estudio de ese tipo de tecnología.

El estudio económico de dicho suceso, incluida la TIC, ha sido abordado en diversos ángulos de investigación, con base en cuatro tipos de exploración: a) como herramienta u objeto técnico - el estudio del desenvolvimiento tecnológico de microcomputadoras, redes, telefonía celular, televisión digital, entre otros-; b) como conjunto de actividades especializadas — comercio y gobierno electrónicos, educación a distancia y salud virtual—; c) como parte de una estrategia de dominio, como puede ser el de mercado por parte de las empresas mediante la publicidad digital, y $d$ ) como parte de un método para la generación del conocimiento, como son las redes digitales de investigación y desarrollo (Linares, 2004).

Desde un enfoque económico, el anterior es un modelo conceptual multivariado en el cual la exploración de la Tic recae frecuentemente en el estudio del conjunto de actividades desarrolladas con el uso de esta tecnología en las sociedades, así como de los beneficios económicos y operativos resultados de sus aplicaciones.

Los esfuerzos teóricos exhiben gran heterogeneidad en aportaciones con un conjunto de estudios de caso que día con día van refutando hipótesis de la frontera internacional del conocimiento y comienzan a construir una base teórica para el estudio del fenómeno tecnológico contemporáneo.

Este artículo tiene el objetivo de brindar elementos para mayor entendimiento de la difusión y del perfeccionamiento del uso de la Tic. Se presentan dos tipos de exploraciones, una teórica y otra contextual. Esta última muestra el caso de dicha difusión y el citado

1 Martin Hilbert y Jorge Katz (2003) señalan que la TIC no es sólo un fruto del desarrollo (por ser consecuencia de éste), sino también, en gran medida, uno de sus motores (por ser una herramienta de desarrollo).

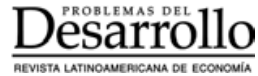


perfeccionamiento en México. La exploración teórica muestra las características generales de la TIC (como objeto técnico), la difusión de la tecnología, la sociedad de la información como imagen de dicha difusión y el perfeccionamiento del uso de la TiC en la creación de oportunidades económicas basadas en la generación y gestión del conocimiento. La finalidad de la exploración teórica es presentar aportes conceptuales actuales y relevantes en una estructura temática coherente y uniforme.

El trabajo se estructura en seis temas. Después de esta introducción, se presenta el contexto de la difusión de la TIC en México en forma narrativa. El tercer tema explora las características generales de la TIC y su difusión; el cuarto aborda aportaciones teóricas acerca de la sociedad de la información, desde los discursos formal (académico y de investigación) y rector (políticas). El quinto tema expone la construcción del concepto economía digital basada en el conocimiento, para aproximarse al estudio del perfeccionamiento del uso de la TIC y aportar a la exploración del surgimiento de los beneficios económicos y operativos de esta tecnología. En el último, se muestran los comentarios finales, desde los dos tipos de exploraciones abordados: contextual y conceptual.

La literatura consultada retoma aportes relevantes de los últimos años en la materia, tanto de organismos internacionales — por ejemplo, la Comisión Económica para América Latina y el Caribe (CEPAL) — , como de esfuerzos teóricos de las corrientes estadounidense de negocios y europea de la gestión del conocimiento y cambio tecnológico.

Este método de compilación de literatura tiene la finalidad de aportar contenidos de la frontera internacional del conocimiento y, con ello, abordar el entendimiento de la exploración de la Tic desde un enfoque económico centrado en la difusión y el perfeccionamiento del uso de la tecnología.

\section{Difusión y perfeccionamiento del uso de la tic en México}

La difusión de la TIC en México, a partir de la informática, inició con el primer equipo de cómputo instalado en 1957 en la Universidad Nacional Autónoma de México (UNAM) y con una fase de inversiones computacionales de la Administración Pública Federal (APF) en los años sesenta para desarrollar el procesamiento electrónico de datos, atendiendo a los requerimientos internacionales de la modernización.

Para alcanzar eficiencia en la asignación de los recursos computacionales en la APF comenzaron a desarrollar lineamientos normativos para racionalizar los recursos informáticos, lo cual derivó en la creación de un sistema de procesamiento electrónico de datos dentro del Programa de Reforma Administrativa del Gobierno Federal (1971-1976). En las décadas siguientes se continuó con los esfuerzos para construir una base de infraestructura informática en la administración pública y para crear un aparato institucional que permitiera el desarrollo nacional en ese ámbito. Se realizaron diversas acciones para la implantación de la política informática, hasta que en mayo de 1996 se publicó el primer Programa 
de Desarrollo Informático (1995-2000), con el objeto de incentivar dicho sector dentro de las áreas económica, social, cultural, académica y legal. ${ }^{2}$

Posteriormente, se planteó la construcción de una plataforma nacional de infraestructura en informática y en medios de comunicación e información, cuyo desenvolvimiento tecnológico derivó, en los años noventa, en la Tic, la cual cobró mayor importancia en las relaciones económicas, políticas y sociales que llevaron a la conformación de un mercado nacional de TIC con constante crecimiento desde finales de los años noventa, que alcanzó en 2002 casi un punto porcentual del mercado mundial. ${ }^{3}$

En ese sentido, el gasto nacional en TIC de 1995 a 2001 mantuvo un incremento promedio anual por arriba de los diez puntos porcentuales, situándose en ese último año en 19 mil millones de dólares (3.2 \% del PІB nacional), apenas por encima del gasto de Brasil de 1995 y muy por debajo de los 137 mil millones de dólares que gastaron en promedio en 2001 países desarrollados como Reino Unido, Alemania y Francia, con gastos entre 8\% y 9\% del PIB. ${ }^{4}$ Estos indicadores demuestran que la inversión de TIC en México aún no es comparable con la de países desarrollados, pero más trascendental es observar que se encuentra por debajo de algunas naciones en vías de desarrollo, determinadas a impulsarse en el desenvolvimiento del nuevo paradigma tecnológico.

En conectividad, en 2002 había en México 82 computadoras por cada mil habitantes (12\% de la densidad computacional de los estados); ${ }^{5}$ en 2003 existían 157 líneas telefónicas por cada mil habitantes (aproximadamente 16\% de la población la tenía); ${ }^{6}$ asimismo, había 1229 usuarios de Internet por cada diez mil habitantes (30\% del promedio de usuarios por cada 10 mil habitantes de los países desarrollados). ${ }^{7}$

En el sector privado no financiero, en 1999, 34\% de los establecimientos contaban con equipo de cómputo; más de $90 \%$ de los de construcción y agroindustria tenían computadora; $44 \%$ de la infraestructura computacional se encontraba en los establecimientos de actividades comerciales; más de $95 \%$ de los grandes establecimientos contaban con infraestructura computacional —observándose una estrecha relación entre su tamaño y el gasto en TIC-, y sólo una tercera parte tenía acceso a Internet. ${ }^{8}$

Plan Nacional de Desarrollo 2001 2006. Programa de Desarrollo Informático.

http://www.inegi.gob.mx/est/contenidos/espanol/tematicos $/$ mediano $/ \mathrm{med} \cdot$ asp?t=tinf $123 \& c=$ 4856 (marzo de 2005).

4 http://WwW.inegi.gob. $\mathrm{mx} / \mathrm{est} / \mathrm{contenidos} / \mathrm{espanol} / \mathrm{tematicos} / \mathrm{mediano} / \mathrm{pai} . \mathrm{asp} ? \mathrm{t}=\mathrm{tinf} 141 \mathrm{\& c}=$ 4869 (marzo de 2005).

5 http://Www.inegi.gob. $\mathrm{mx} / \mathrm{est} / \mathrm{contenidos} / \mathrm{espanol} / \mathrm{tematicos} / \mathrm{mediano} / \mathrm{pai} . \mathrm{asp} ? \mathrm{t}=\mathrm{tinf} 144 \& c=$ 4872 (marzo de 2005).

${ }^{6}$ http://WWw.inegi.gob.mx/est/contenidos/espanol/tematicos $/$ mediano/pai.asp?t=tinf $140 \& c=$ 4868 (marzo de 2005).

7 http://Www.inegi.gob.mx/est/contenidos/espanol/tematicos/mediano/pai.asp?t=tinf $142 \& c=$ 4870 (marzo de 2005).

8 http://www.inegi.gob.mx/est/default.asp?c=3447 (marzo de 2005).

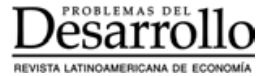




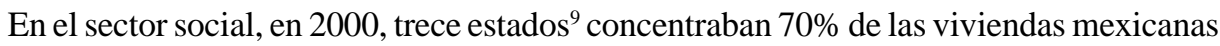
con computadora, $66 \%$ con teléfono y $53 \%$ con televisión; ${ }^{10}$ en 2002 sólo $1 \%$ de los hogares con ingresos entre cero y cuatro salarios mínimos contaban con computadora, mientras que $65 \%$ con mayores ingresos (superiores a los 20 salarios mínimos) tenía al menos una: ${ }^{11}$ y en 2004 , de los 26.3 millones de hogares mexicanos $92 \%$ tenía televisión, $48 \%$ teléfono, $18 \%$ computadora y $9 \%$ conexión a Internet. ${ }^{12}$

En 2004, 25\% de la población (mayores a los seis años de edad) usaba computadoras; $14 \%$ utilizaba Internet; los hogares, el trabajo y la escuela eran los principales lugares de acceso a las máquinas, para apoyo escolar, funciones laborales y entretenimiento, respectivamente; las principales aplicaciones computacionales empleadas eran los procesadores de texto, hojas de cálculo y manejadores de bases de datos; la población que hacía mayor uso de una computadora e Internet se encontraba entre 12 y 34 años de edad y los programas de Internet más utilizados eran para correo electrónico, consulta en línea y chat. ${ }^{13}$

Finalmente, en la administración pública, en 2000, el manejo principal de Internet era la prestación de servicios y atención a la población, desarrollo de redes institucionales, control de TIC y monitoreo remoto de equipo; ${ }^{14}$ y en 2001 , en promedio, $98 \%$ de las dependencias contaban con computadoras personales, ${ }^{15}$ de las cuales 74 de cada 100 estaban conectadas a red local. ${ }^{16}$

Esta penetración de las tecnologías digitales en las estructuras económicas, políticas y sociales del país ha ido de la mano de la formación del capital humano que, asimismo, ha ido en constante crecimiento, consolidándose como uno de los grupos de programas académicos de nivel técnico, superior y de posgrado con mayor demanda. En el ciclo escolar 2001-2002 los programas de nivel superior en TIC registraron una matrícula nacional de 177110 alumnos inscritos, es decir, se ubicaron en la segunda plaza de mayor demanda académica del país, tan sólo después de derecho, y en cuanto a la distribución estatal, estaba concentrada en siete entidades federativas con poco más de 50\%: Distrito Federal

9 Aguascalientes, Baja California, Baja California Sur, Coahuila, Colima, Chihuahua, Distrito Federal, Jalisco, México, Nuevo León, Querétaro de Arteaga, Quintana Roo y Sonora.

10 http://Www.inegi.gob.mx/est/default.asp?c=3454 (Mar 2005)

11 http://www.inegi.gob.mx/est/contenidos/espanol/tematicos $/$ mediano/med.asp?t=tinfo38\&c= 3462 (marzo de 2005).

12 http://Www.inegi.gob.mx/est/contenidos/espanol/tematicos $/$ mediano/med.asp?t=tinf $196 \& c=$ 5585 (marzo de 2005).

13 http://Www.inegi.gob.mx/est/default.asp?c=3454 (Mr 2005)

14 http://Www.inegi.gob.mx/est/contenidos/espanol/tematicos/mediano/med.asp?t=tinfo $13 \& c=$ 3435 (marzo de 2005).

15 http://Www.inegi.gob. $\mathrm{mx} / \mathrm{est} / \mathrm{contenidos} / \mathrm{espanol} / \mathrm{tematicos} / \mathrm{mediano} / \mathrm{med} \cdot \mathrm{asp} ? \mathrm{t}=\mathrm{tinfoO} 1 \& \mathrm{c}=$ 3423 (marzo de 2005).

16 http://Www.inegi.gob. $\mathrm{mx} /$ est/contenidos/espanol/tematicos/mediano/med.asp?t=tinfoO2\&c= 3424 (marzo de 2005).

\section{DeSarrollo}


(15\%), Estado de México (10\%), Veracruz (8\%), Tamaulipas y Jalisco (6\%), Puebla (5\%) y Nuevo León (4\%). ${ }^{17}$

Respecto de los principios rectores, en los últimos años se ha comenzado a conformar un marco legal y otro normativo para reglamentar las acciones nacionales informacionales, incluyendo actividades de digitalización. En esa línea se han creado —en el nivel nacionalla Ley Federal de Transparencia y Acceso de la Información Pública Gubernamental, la Ley de Información Estadística y Geográfica y la Ley para Regular las Sociedades de Información Crediticia, así como una serie de normatividades internas —en los niveles organizacional e institucional- que regulan las acciones de digitalización al interior de las organizaciones, las cuales están relacionadas con procesos informacionales y operativos, como son los reconocimientos de la firma e identificación electrónicas en transacciones comerciales, en diversos ámbitos de competencia. Con ello, dichos principios están siendo guiados hacia las siguientes acciones y disposiciones:

- Promover lo necesario para que toda persona pueda tener acceso a la información mediante procedimientos sencillos y expeditos.

- Transparentar la gestión pública por medio de la difusión de la información.

- Garantizar la protección de datos personales.

- Mejorar la organización, clasificación y manejo de documentos.

- Normar el funcionamiento de los servicios nacionales de información estadística y geográfica.

- Regular la difusión del uso de los medios electrónicos en las actividades comerciales.

- Implantar el uso de identificaciones electrónicas en los ámbitos administrativos, económicos y organizacionales de competencia, entre otros.

Ante ese contexto nacional, la APF de México, como en el resto del mundo, ${ }^{18}$ ha retomado las riendas de la dirección y el control de la difusión de la TIC en la sociedad, con el enfoque de la construcción de la sociedad de la información. La estrategia principal es integrar las acciones digitales de los agentes nacionales en un sólo esfuerzo común.

La APF ha desarrollado el proyecto digital e-México, propuesta del Ejecutivo Federal para construir una sociedad de la información de "alto valor ciudadano" con un modelo gobierno a ciudadano. La propuesta intenta tomar en cuenta e integrar los resultados del crecimiento y el desarrollo de la difusión de la Tic en México, con el objetivo de generar contenidos sociales que ofrezcan herramientas y oportunidades para mejorar la calidad

17 http://www.inegi.gob.mx/est/default.asp?c=3465 ((marzo de 2005).

18 Véase e Governmnet en Europa: The State of Affairs (2004); declaración presidencial acerca de la promulgación de la Ley sobre el Gobierno Electrónico de los Estados Unidos de Norteamérica (17 de diciembre de 2002), y CEPAL (2003).

\section{DeSarrollo}


de vida de los mexicanos. La propuesta digital está organizada en tres grandes ejes: conectividad, ${ }^{19}$ contenidos ${ }^{20}$ y sistema. ${ }^{21}$

Los propósitos del proyecto e-México son de carácter eminentemente social debido a que promueve la conectividad y generación de contenidos digitales mediante Internet con el fin de apoyar la integración poblacional en el desarrollo económico y social de México, intentando reducir la brecha de conectividad con la creación de centros comunitarios digitales (CCD) y la brecha digital por medio de la capacitación de las familias mexicanas en el uso de la TIC y difusión del conocimiento; líneas de acción que ponen a disposición de la población la información y los servicios que prestan los gobiernos federal, estatal y municipal.

En síntesis, la construcción de la sociedad de la información en México es el proceso integrador de la difusión de la TIC en el país. Actualmente, está en una fase de implantación y los resultados arrojados en los próximos años llevarán a nuevos desarrollos. La meta es alcanzar la maduración y consolidación en este proceso tecnoeconómico y, a la postre, desarrollar una economía digital basada en el conocimiento.

Por lo antes mencionado, en todos los niveles sociales son: $a$ ) la brecha doméstica de conectividad —en lo inmediato—, b) la brecha doméstica digital —en el mediano plazo— - y c) la brecha digital internacional —en el largo plazo—, en la cual el bajo poder adquisitivo —-medio poblacional—, la concentración regional del desenvolvimiento tecnoeconómico de las tecnologías digitales y la falta de capacidades digitales nacionales —conocimientos, habilidades y experiencia— son las principales problemáticas.

A continuación se exponen algunas aportaciones teóricas de los últimos años que han permitido el estudio de la difusión de la Tic en las sociedades. Además, se expone la construcción del concepto economía digital basada en el conocimiento, para vincular la difusión de la tecnología con aspectos económicos relacionados con la teoría de la gestión del conocimiento. Al final, ambas líneas conceptuales convergen hacia un enfoque económico integral que permite estudiar la TIC a partir de la exploración de la difusión y del perfeccionamiento del uso de la tecnología en las sociedades.

19 La conectividad se recarga en el hecho de incrementar las inversiones en infraestructura física de telecomunicaciones, así como en la creación de una red de centros digitales que sirvan para brindar conectividad a las poblaciones y familias que, por limitaciones económicas y geográficas, no cuentan con dicha infraestructura para integrarse a la naciente sociedad nacional de la información.

20 Los contenidos digitales se agrupan en cinco sectores verticales o sistemas digitales de información: e aprendizaje, e salud, e economía, e ciencia, tecnología e industria y e -gobierno.

21 Los sistemas se enfocan en la creación de un portal de portales, un punto neutral de acceso a la red (NAP) y un centro de cómputo del gobierno como los tres puntos principales por desarrollar, mediante los cuales se ofrecerán los servicios y productos públicos digitales y se realizarán las actividades de modernización del Estado mexicano. Véase www.e-mexico.gob.mx (marzo de 2005).

\section{DeSarrollo}




\section{La tecnología de la información y las comunicaciones (тіс)}

La TIC, como parte del fenómeno tecnológico contemporáneo, puede ser explorada por medio de cuatro modalidades: $a$ ) herramienta u objeto técnico; $b$ ) mecanismo de aprendizaje y de generación de conocimientos; $c$ ) un conjunto de actividades especializadas; y d) medio para ejercer una voluntad colectiva de dominación y control (Linares, 2004).

Como objeto técnico, en la construcción de la sociedad de la información (Hilbert y Katz, 2003), la CEPAL identifica a la TIC como la convergencia entre las trayectorias tecnológicas de los medios de información, de comunicación y de informática: Internet, telefonía celular 3G, televisión digital y asistentes digitales personales. Se define como un sistema tecnológico conformado por cuatro componentes básicos (Hilbert y Katz, op. cit.): a) infraestructura física, $b$ ) software, $c$ ) estructuras de comunicaciones y mecanismos de coordinación, ${ }^{22}$ y $d$ ) contenidos. Su uso permite que las personas puedan comunicarse y compilar, generar, manejar, transferir y divulgar información desde puntos geográficos distantes, por medio de desarrollo de hardware y software especializados.

El resultado de la utilización de la TIC conlleva a la digitalización de datos, información y conocimientos en formatos digitales de texto, imágenes, sonidos, voz u otros, los cuales son almacenados, manejados, procesados y difundidos en el denominado proceso informacional. ${ }^{23}$

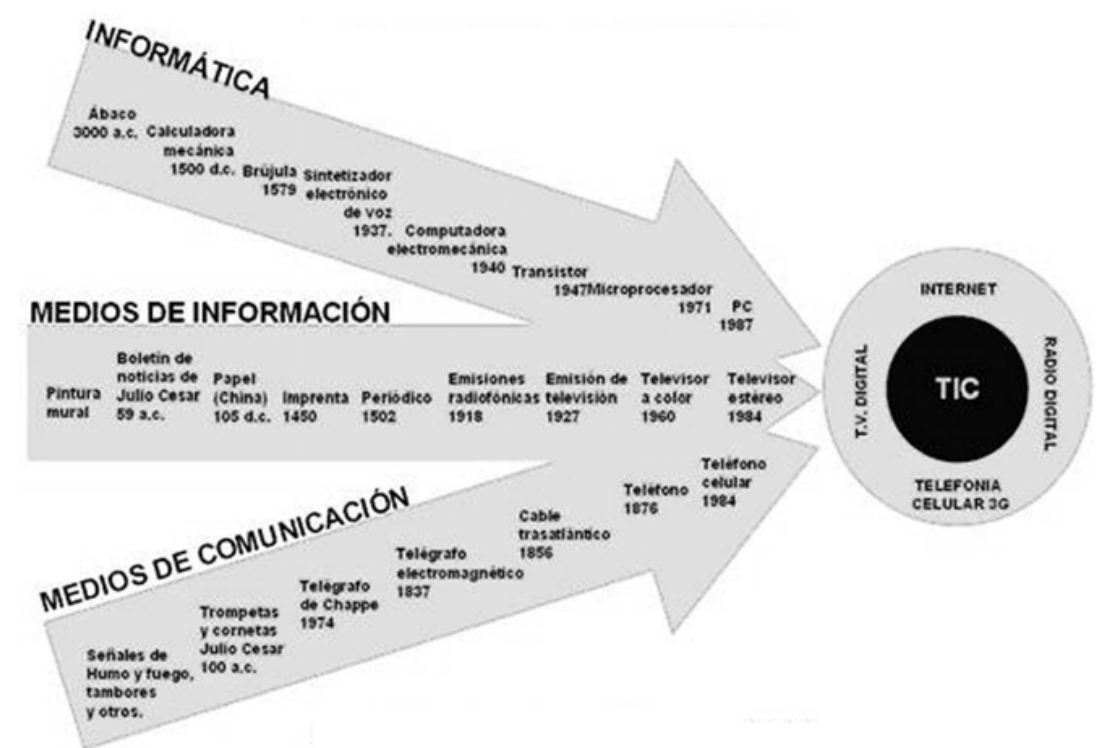

Gráfica 1. Trayectorias tecnológicas de la informática y de los medios de información y comunicación. Fuente: elaborado con base en Hilbert y Katz (ibid.).

22 Las formas en las cuales se estructura, organiza y maneja la información.

23 En este ensayo, el proceso informacional se define como el conjunto de acciones que permiten generar información a partir de un conjunto de datos existentes, cubriendo los requerimientos 
Con la creciente demanda de información y de conocimientos de los agentes para el desarrollo de sus funciones y, en particular, para la toma de decisiones, se ha potenciado la difusión de la TIC, debido a que el conjunto de estas acciones pueden realizarse mediante sus aplicaciones.

A partir de este comportamiento, la difusión de la TIC se ha incrementado por medio del desarrollo de actividades especializadas como el comercio y el gobierno electrónicos, que han provocado nuevas formas o arreglos sociales y productivos, comúnmente referidos como sociedad de la información. Con ello, la información y el conocimiento digitales se han convertido en elementos de eficiencia y competitividad para las empresas, instituciones y organizaciones (Castells, 1996).

A la fecha, Internet es la TIC más aceptada debido a que permite mayor interconexión de aplicaciones digitales ${ }^{24}$ mediante redes, brinda acceso público a contenidos digitales ${ }^{25}$ estructurados en sitios y portales, ofrece oportuna transferencia de datos, información y conocimientos previamente digitalizados, y promueve la exactitud y relevancia en sus contenidos mediante acciones presentes y futuras que contrarrestan la infección de la información por virus digitales, la desinformación y la no usabilidad ${ }^{26}$ de los portales.

Su difusión se inició principalmente en empresas trasnacionales con dos enfoques de aplicación (Kogut, 2003a): a) la transmisión de contenidos por medio de la red y b) la explotación de las externalidades positivas; ${ }^{27}$ propiciando en corto tiempo (diez años) un proceso de difusión global.

En los ámbitos organizacionales y nacionales, la difusión de Internet está estrechamente relacionada con dos bloques de elementos que facilitan su difusión, los cuales están dirigidos a la búsqueda incesante de mayor difusión y más eficiencia (Kogut, 2003b; Kenney, 2003; Glimstedt y Zander, 2003; Benghozi y Licoope, 2003; Zaheer y Rajan, 2003; Casper, 2003; Chang, 2003; Sako, 2003):

informacionales del demandante. Para mayor entendimiento véase Murdick y Munson (1988) y Burch y Grundnitski (1989), entre otros.

24 En este artículo son los servicios genéricos que ofrece este sistema tecnológico a los usuarios tales como: correo electrónico, mensajes de voz, transferencia electrónica de datos, comunicación telefónica en línea, buscadores web, entre otros.

25 Se entiende como el conjunto de datos, informaciones y conocimientos digitalizados por medio de la utilización de TIC e infraestructura informática.

26 Medida en la cual un producto o servicio puede ser usado para conseguir objetivos específicos con efectividad, eficiencia y satisfacción, en cierto contexto (ISO 9241 11, 1998). Está conformada por facilidad de aprendizaje, eficiencia en su uso, memorabilidad y minimización de la frecuencia y severidad de errores. En el medio digital, un sitio usable debe considerar los siguientes puntos: aproximación al usuario, amplio conocimiento del contexto del uso, determinación de la usabilidad (INFOTEC en Punto Gob, enero de 2002).

27 A medida que se incrementa el número de usuarios conectados a Internet, el valor de la red crece debido a que potencialmente sería mayor la penetración en los mercados (Kogut, 2003a).

\section{DeSarrollo}


Cuadro 1

Internet como sistema tecnológico

\begin{tabular}{|c|c|}
\hline Capas o Plataformas tecnológicas & Ejemplos \\
\hline Webs & Portales y páginas comerciales, gubernamentales, educativas \\
\hline Aplicaciones & Procesadores de texto, aplicaciones de productividad. \\
\hline Medias & Browsers, sistemas de administración de bases de datos \\
\hline \multicolumn{2}{|l|}{ Software de interfase: } \\
\hline Estandarización de información y protocolos & HTML,XML,WAP \\
\hline Direcciones y rutas & Dominios y http \\
\hline Protocolos de datos & $\mathrm{TCP} / \mathrm{IP}$ \\
\hline Sistemas operativos & Unix, Linux, Windows \\
\hline \multicolumn{2}{|l|}{ Hardware: } \\
\hline Backbone & Conmutadores, líneas de fibra óptica, estaciones satelitales \\
\hline Servers & Plataforma computacional \\
\hline Dispositivos móviles eventuales & PDA, Telefonía móvil \\
\hline
\end{tabular}

Fuente: Feldman(1999) y Kogut (2003a).

- En el ámbito organizacional, por los modelos tecnológicos adoptados, políticas implantadas y la cultura organizacional. ${ }^{28}$

- En el ámbito nacional, por la infraestructura tecnológica, la difusión del conocimiento tecnológico, el capital financiero de riesgo, los precios de los proveedores de Internet, la apertura comercial y la trayectoria de la estrategia nacional de utilización de dicha red.

Desde un enfoque técnico, Internet se define como un complejo sistema tecnológico digital de información y comunicación que relaciona cuatro subsistemas esenciales: 1) infraestructura física, 2) terminales y servicios, 3) protocolos de interfase, y 4) aplicaciones tecnológicas (servicios genéricos).

La difusión de Internet se da en el plano de los sistemas de información ${ }^{29}$ mediante sistemas de procesamiento de transacciones. ${ }^{30}$ Con su uso, empresas, instituciones y orga-

28 La cultura organizacional se identifica, en el presente artículo, como la forma en la cual la organiza ción hace las cosas para el desarrollo de acciones concretas, por lo cual la cultura organizacional se muestra como el comportamiento funcional de la organización, caracterizado por el conjunto de conductas, capacidades, habilidades, conocimientos, experiencias y jerarquías organizacionales - elementos de la cultura organizacional.

29 Los sistemas de información están conformados por un grupo de agentes que interactúan entre sí durante el proceso informacional, en el cual la transferencia de datos y de información se realiza por medio de las capacidades sensoriales humanas, medios de información y comunicación y herramientas digitales. Los principales retos en la construcción de sistemas de información organizacionales son la oportuna transferencia, la exactitud y la relevancia de contenidos (Bruch y Grundnitski, 1989).

30 Los sistemas de procesamiento de transacciones son aquellos que sustituyen los procesos manuales por otros basados en herramientas informáticas - y TIC - (Senn, 1992).

\section{DeSarrollo}


nizaciones intentan alcanzar ventajas competitivas y eficiencia económica en los diversos ámbitos de competencia, por medio de la aplicación de los conocimientos, habilidades y experiencias organizacionales en la construcción de sistemas digitales de información (Dodgson, Gann y Salter, 2001; Fine y Raff, 2000).

La utilización de Internet en las sociedades dio lugar a la construcción de sistemas digitales de información estructurados en los denominados sitios y portales, los cuales agrupan un conjunto de datos, información y conocimientos tipificados, ya sea por agentes económicos (empresa, gobierno y ciudadanos) o por temas específicos (democracia, cultura, aprendizaje, por mencionar algunos).

Cada grupo de contenidos en Internet se identifica con el prefijo $e$-, el cual evidencia que parte o el total de los contenidos de un agente económico o de un tema particular han sido digitalizados y están expuestos en un portal o página web particular: e-gobierno, e-comercio, e-salud, entre otros.

Estos sistemas digitales están estructurados en modelos económicos de interacción de contenidos entre los diversos agentes, conformando la naciente economía digital, cuya base es el conocimiento. Cada modelo implica una relación proveedor-usuario específica en la transferencia de contenidos y mantienen una estructura sistémica, la cual puede desarrollarse de forma abierta o cerrada con respecto de su entorno, mediante relaciones permanentes o temporales con cierto grado de formalidad e informalidad en función del trato económico-social. Entre los modelos de mayor relevancia actual tenemos:

- $\mathrm{B} 2 \mathrm{~B}-$ Business to business (negocio a negocio)-;

- $\mathrm{B} 2 \mathrm{C}$-Business to commerce (negocio a comercio y a consumidor)—;

- $\mathrm{G} 2 \mathrm{G}$ - Government to government (gobierno a gobierno)-;

- G2B - Government to business (gobierno a negocio)—; y

- $\mathrm{G} 2 \mathrm{C}$-Government to citizens (gobierno a ciudadano)—, entre otros.

Actualmente, la difusión de Internet es la temática que suscribe el estudio de la sociedad de la información; en particular se explora el acceso a la tecnología, el crecimiento de la infraestructura tecnológica que conforma este sistema digital y el crecimiento de los contenidos y sistemas de información comprendidos en las páginas y portales digitales.

\section{Sociedad de la información}

Como se mencionó en párrafos anteriores, la difusión de la TIC está permitiendo nuevas formas de arreglos sociales y productivos. En los últimos años, su estudio ha sido abordado más exhaustivamente a partir de dos tipos de discurso: 1) el formal —académico y de investigación- y 2) el rector — político—, que identifica la construcción de la sociedad de la información como un proceso estratégico guiado por las administraciones públicas

\section{DeSarrollo}


Cuadro 2

Sistemas de información y modelos de utilización de Internet

\begin{tabular}{lccccc}
\hline & & E-Comercio & E-Gobierno & E-Educación & E-Sociedad \\
\hline & $\begin{array}{l}\text { Proveedor } \\
\text { /Usuario }\end{array}$ & Empresas & Gobierno & $\begin{array}{c}\text { Instituciones } \\
\text { académicas }\end{array}$ & Ciudadanos \\
\hline E-Comercio & Empresa & B2B & B2G & B2E & B2C \\
E-Gobierno & Gobierno & G2B & G2G & G2E & G2C \\
E-Educación & $\begin{array}{l}\text { Instituciones } \\
\text { académicas }\end{array}$ & E2B & E2G & E2E & E2C \\
E-Sociedad & Ciudadanos & C2B & C2G & C2E & C2C \\
\hline \begin{tabular}{l} 
Sistemas de información \\
Agentes fuentes \\
\hline Modelos de integración
\end{tabular} & $\begin{array}{l}\text { B: Business (negocios) } \\
\text { G: Government (gobierno) }\end{array}$ & $\begin{array}{l}\text { E: Education (educación) } \\
\text { C: Citizens (ciudadanos) }\end{array}$
\end{tabular}

Fuente: elaborado con base en diversas aportaciones literarias.

que se encargan del diseño, implantación y regulación de la integración y expansión de las actividades digitales.

El discurso formal parte de la observación de que este tipo de sociedad es aún incipiente ${ }^{31}$ con la connotación que diferencia a los países desarrollados de los menos desarrollados, encontrándose esta línea de investigación en un proceso de catch-up (aprendizaje y entendimiento), en el cual las hipótesis se generan y confirman por medio de diversos esfuerzos e interpretaciones de las experiencias del uso de dichas tecnologías en empresas, instituciones y organizaciones.

Algunos esfuerzos teóricos que abordan esta temática se basan en la exploración económica de las características de la Tic y en el proceso de digitalización resultante de su uso, como es el modelo de construcción de la sociedad de la información propuesto por la CEPAL. En esta línea conceptual, la CEPAL define la sociedad de la información como el conjunto de agentes económicos que han iniciado acciones de digitalización ${ }^{32}$ por medio del uso de la infraestructura informática y de la TIC, pero sobre todo por medio de las aplicaciones tecnológicas de Internet (Hilbert y Katz, op. cit.). Subraya que la construcción de la sociedad de la información requiere de la difusión de la Tic, de la informática y

31 La difusión comercial y aplicación internacional de la TIC data de finales de los años ochenta, cuando se genera en Estados Unidos el embrión de lo que actualmente es Internet por medio de la fusión de ARPANET, NSFNET y otras redes de libre acceso. Ante esa evidencia, se habla de aproximadamente veinte años desde el surgimiento y difusión masiva de esas tecnologías, y se observa a la fecha una constante generación de invenciones incrementales acerca de este paradigma tecnológico, situación que demuestra que el desenvolvimiento tecnológico está aún en sus primeras fases.

32 Se refieren a la digitalización de contenidos, sistemas de información, comunicaciones y mecanismos de coordinación de individuos, empresas, organizaciones e instituciones de la administración pública. 
de las telecomunicaciones en las actividades informacionales de las esferas operativas y económicas de los agentes.

La CEPAL observa que para edificar una sociedad de la información, a partir de la difusión de la TIC, es indispensable estructurar dicha construcción con elementos que soporten el proceso. Desde este enfoque, Hilbert y Katz proponen un modelo apoyado en la articulación de cuatro elementos básicos mediante los cuales se le da forma:

- Infraestructura tecnológica digital

- Capital humano

- Capital financiero

- Marcos normativos

La infraestructura informática comprende la existencia e innovaciones en hardware y software, que son el soporte del proceso de construcción de la sociedad de la información debido a que el desarrollo tecnológico digital es el elemento indispensable para la realización de acciones (Hilbert y Katz, ibid.). La eficiencia y rentabilidad dependen de las mejoras incrementales y radicales.

Para que las acciones de digitalización y el desenvolvimiento del nuevo paradigma tecnológico (Pérez, 1986) envuelvan al mayor número de agentes de una sociedad, se requiere de elementos complementarios que se articulen entre sí y con la infraestructura tecnológica para generar productos y procesos digitales.

El capital humano requerido para el desarrollo de las acciones digitales es aquel que ha formado conocimientos, habilidades y experiencias en el uso de estas tecnologías en funciones operativas e informacionales, a partir de su incorporación a diversos mecanismos de aprendizaje tecnológico, ${ }^{33}$ derivados de esfuerzos individuales y colectivos. ${ }^{34}$

En las acciones de digitalización, el aprendizaje toma un comportamiento cíclico, integral y complementario que inicia con un proceso de aprendizaje en cuanto a la utilización de tecnologías de vanguardia y continúa con los usos de la aplicación digital en funciones operativas y con la creación de nuevos desarrollos tecnológicos (Lira, 2005).

33 El aprendizaje tecnológico es el esfuerzo o proceso organizacional para conocer o saber cómo asimilar y adoptar nuevas tecnologías en las operaciones económicas de la organización, es decir es el proceso que se realiza al interior de la organización para adquirir conocimientos o saberes acerca de nuevas tecnologías con la finalidad de permitir su asimilación y adaptación organizacional. Algunos de los principales mecanismos de aprendizaje en las organizaciones son: learning by doing (aprender haciendo), learning by working (aprender trabajando) learning by trail and mistake (aprender por ensayo y error), learning by train (aprender por capacitación) y la reingeniería de reversa, entre otros.

34 Los procesos organizacionales de aprendizaje tecnológico se pueden desarrollar en los niveles individual y colectivo mediante los esfuerzos internos de los miembros de la organización o por la incorporación de nuevos miembros (Dutrenit, 2000).

\section{DeSarrollo}


El reto operacional del capital humano es alcanzar el dominio de la tecnología en los procesos establecidos (Villavicencio, 1989) y conformar así una base de capacidades tecnológicas ${ }^{35}$ que permitan enfrentar los retos y problemas que se vayan suscitando, así como el alcance de beneficios económicos.

El capital humano es elemento indispensable para la difusión de tecnologías digitales y el motor de la construcción de la sociedad de la información, a partir del crecimiento de las acciones de digitalización y de sus procesos y productos resultantes (Lira, op. cit.).

Las fuentes de financiamiento son un elemento indispensable para el desarrollo, implantación y continuidad de la digitalización social. Cada proyecto en sí mismo requiere de capital financiero para su desarrollo, particularmente en lo referente a la adquisición de infraestructura tecnológica (Hilbert y Katz, 2003).

Por su parte, los marcos normativos son el elemento complementario que facilita la difusión de la TIC y la construcción de la sociedad de la información. Hilbert y Katz (ibid.) mencionan que el accionar de éstos tienen el objetivo de organizar, controlar y regular las acciones de digitalización emprendidas por la sociedad.

Un marco normativo flexivo y efectivo permite una mejor y más eficiente realización y desarrollo de la difusión del uso social de Internet y tecnologías digitales (Lira, ibid.). Sin embargo, para alcanzar mayor eficiencia en la difusión de la TIC en las diversas esferas nacionales e internacionales y en la construcción de la sociedad de la información es indispensable el diseño y establecimiento de estrategias digitales ${ }^{36}$ que guíen el proceso de desenvolvimiento tecnológico a partir de la articulación de los elementos de difusión de estas tecnologías (Lira, ibid.).

Las experiencias internacionales en política digital muestran que las estrategias son diseñadas por niveles — micro, meso y macro- en función de las características particu-

35 Una forma de conceptuar las capacidades tecnológicas es como la habilidad propia de hacer uso efectivo del acervo de conocimiento tecnológico para asimilar, usar, adaptar y cambiar las tecnologías existentes (Westphal, Kim y Dahlman, 1985; Bell y Pavitt, 1995; Kim, 1997; Leonard, 1992 y 1995). Y, en un sentido más amplio, es la acción de hacer uso efectivo del conjunto de habilidades, experiencias, acervos de conocimiento, cultura y rutinas propias de la organización para afrontar los cambios tecnológicos existentes por medio de procesos de aprendizaje tecnológico.

36 Cuando una tecnología específica se convierte en el centro corporativo, económico y en uno de los mayores recursos de ventaja competitiva para las organizaciones, las estrategias tecnológicas toman gran realce, porque permiten: a) comprender y enfrentar la incertidumbre, complejidad y disconti nuidad de los cambios tecnológicos, $b$ ) observar el contexto global de la tecnología, c) atender com plementariedades entre las estrategias corporativas y entre las de las compañías, $d$ ) identificar los puntos débiles por los cuales la tecnología no logra integrarse exitosamente y e) relacionar la es trategia tecnológica corporativa y las políticas tecnológicas gubernamentales (Dodgson, 1991). Las estrategias de tecnologías de información se suscriben generalmente en las estrategias digitales, cuyo principal objetivo es hacer de estas herramientas la llave de las competencias centrales de las organizaciones (Andreu y Valor, 2004; Ciborra, 1994; Earl, 1994), creando nueva información y conocimientos (Nonaka, 1988) dirigidos a una finalidad económica clara: crear valor (Rieck y Dickson, 1993; Ciborra, 1994).

\section{DeSarrollo}


lares de cada región y cultura digital establecida, con diversas modalidades de exposición. De igual modo, señalan la necesidad de un enfoque dinámico que permita incluir las innovaciones tecnológicas, así como los cambios de conducta en la cultura digital, haciéndose indispensable la integración del mayor número de agentes y conceptos e ideas posibles, ya que ese ángulo permite un desenvolvimiento tecnoeconómico-social envolvente (Lira, ibid.).

Ante ese conjunto de aportaciones teóricas, la sociedad de la información se define como el proceso estratégico de interconexión entre infraestructura tecnológica digital, los capitales humano y financiero y los marcos normativos para la realización de acciones, cuyo resultado da lugar a la digitalización de contenidos y de sistemas de información, comunicaciones y mecanismos de coordinación de un conjunto de sectores sociales (negocios, gobierno, salud, educación, entre otros) o de un grupo de áreas conceptuales (democracia, aprendizaje, cultura, por mencionar algunos).
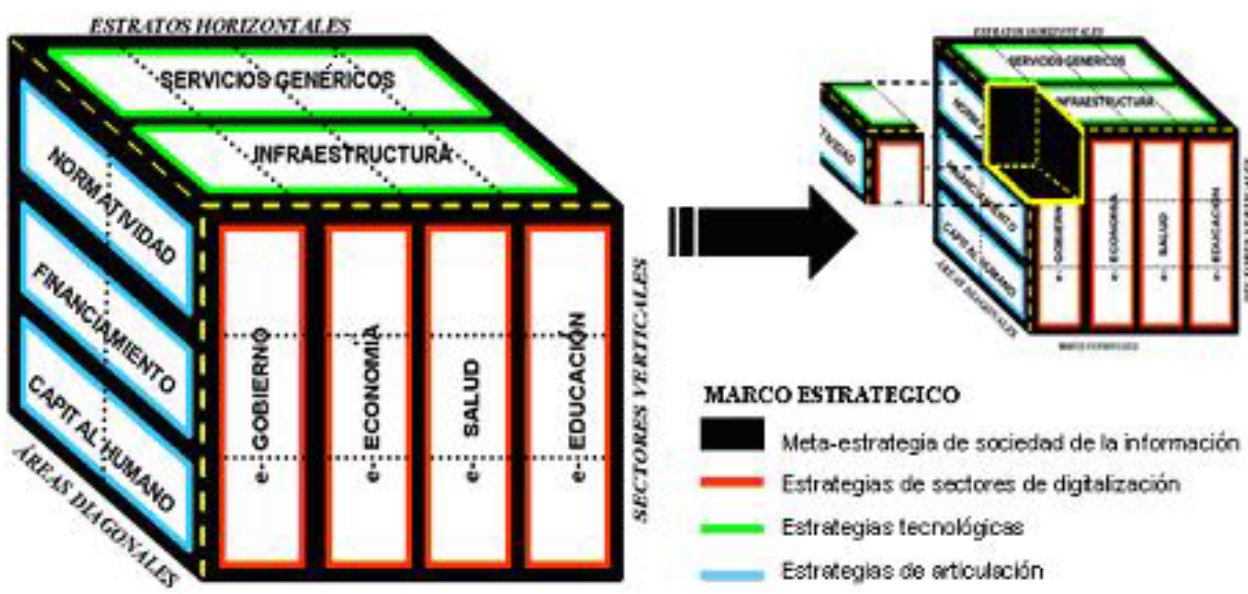

Gráfica 2. Elementos para la construcción de la sociedad de la información.

Fuente: elaborado con base en Hilbert y Katz, 2003.

Este enfoque conceptual permite observar las áreas temáticas que se deben involucrar y las respectivas responsabilidades de los agentes implicados, tanto para acrecentar el acceso a la TIC, como para incrementar el número de prácticas digitales y, con ello, construir una sociedad de la información a partir del diseño y establecimiento de una estrategia integral.

En este proceso, la infraestructura — hardware y software — constituye la plataforma técnica y el desenvolvimiento tecnológico; los marcos normativos, fuentes de financiamiento y el capital humano, los elementos que facilitan la difusión de la TIC en las sociedades, y los productos, servicios y procesos digitales, generados por los agentes, el crecimiento de la sociedad de la información en una región. 
Finalmente, el discurso formal denota que debido a la diversidad de condiciones sociodemográficas en las regiones, no existe una solución única hacia la transición a la sociedad de la información (Hilbert, Bustos y Ferraz, 2003).

En el discurso rector (políticas) se observa que los objetivos de la conformación de una sociedad de la información se sustentan en una estrategia gubernamental que subraya el compromiso internacional de construir dichas sociedades centradas en las personas, integradoras y orientadas al desarrollo nacional, en las cuales los gobiernos, empresas, organizaciones y sociedad civil sean los agentes que encaucen el potencial de las TIC hacia la promoción de objetivos de desarrollo. ${ }^{37}$

No obstante, la construcción de una sociedad de la información conlleva a una relación de desvinculación entre los agentes que salieron del proceso y los que se integran a las actividades de digitalización, fenómeno comúnmente referido en dos connotaciones: a) como brecha de conectividad, y b) como brecha digital.

La brecha de conectividad es el grado de acceso a la tecnología más avanzada de información y comunicación e implica la capacidad de penetración de la TIC en las regiones y naciones (brecha internacional de conectividad) y en los grupos ciudadanos de una sociedad (brecha doméstica de conectividad).

La brecha digital, por su parte, involucra la capacidad de procesamiento de información y la creación de redes que beneficien a todos y puedan contribuir a elevar las condiciones de vida de una sociedad (Cv Mistica, 2002) (Hilbert y Katz, op. cit.); interpretada como el abismo que separa a la regiones y a los países (brecha digital internacional) y a los grupos ciudadanos de una sociedad (brecha digital doméstica) debido a que denota el grado de integración de una sociedad de la información.

Ambas connotaciones están relacionadas con variables económicas vinculadas con el desenvolvimiento de las tecnologías digitales, por lo cual han sido utilizadas como indicadores de crecimiento económico en los entornos internacionales (CEPAL, 2000 y 2003); por ejemplo, el acceso a Internet y la adquisición de infraestructura tecnológica digital han sido asociados con la medición del crecimiento económico por su estrecha relación con el ingreso per capita, y también algunas de las principales causas que inhiben el uso y desenvolvimiento de la $\operatorname{TIC}^{38}$ han sido empleadas como manifestación económica de las sociedades: la inadecuada infraestructura tecnológica digital y el bajo desarrollo de servicios genéricos (software).

Ante esas implicaciones y ante la relevancia internacional de Internet, a finales de los años noventa, Estados Unidos retomó el uso del concepto brecha digital para denotar el riesgo

37 Cumbre Mundial sobre la Sociedad de la Información, Ginebra 2003 Túnez 2005. Documento WSIS 03/GENEVA/4s, mayo de 2004. Declaración de Principios. Construir la Sociedad de la Información: Un desafío global para el nuevo milenio.

38 Falta o inadecuada infraestructura en TIC, bajo desarrollo de servicios genéricos (software), falta de flexibilidad institucional, bajos niveles de ingreso y académico, barreras idiomáticas, uso inadecuado de contenidos, edad y género (Kogut, 2003b; Katz y Hilbert, ibid.; Cecchini, 2004).

\section{DeSarrollo}


de marginar a los sectores sociales menos favorecidos de los beneficios de Internet, con la connotación de que ésta es la puerta de entrada al mundo de la información y del conocimiento (Volkow, 2003). Con ello, el grado de construcción de una sociedad de la información ha sido comúnmente referido como el grado de conectividad a Internet - como indicador de acceso- y como la capacidad de procesamiento de contenidos digitalizados en la red —difusión de Internet- como indicador de desenvolvimiento tecnológico.

Finalmente, la sociedad de la información, en el discurso rector, es un sistema social de información y comunicaciones construido por medio de la digitalización de contenidos con el uso de Internet. Los contenidos son estructurados por temas de interés o por agentes. Con ello, los sitios y portales son expresión de los esfuerzos y prácticas digitales de la sociedad de la información en una región.

La finalidad de dicha sociedad y de las prácticas digitales es alcanzar mayor eficiencia en el desarrollo de las mismas actividades, mejorar los sistemas de información y comunicaciones, públicos y privados, para la toma de decisiones y obtener beneficios económicos por medio de ahorro en procesos operativos y de la apertura de oportunidades económicas en los mercados o ámbitos de competencia.

Para alcanzar esos resultados, la utilización de la tecnología debe ser perfeccionada en el conjunto de actividades digitales especializadas, para hacer de la difusión tecnológica un medio de generación de conocimientos, invenciones e innovaciones, como se expone a continuación.

\section{Economía digital basada en el conocimiento}

La difusión de la TIC e Internet ofrece el potencial de la obtención de beneficios económicos y operativos, debido a que la información se ha convertido en bien económico mediante el cual se realizan actividades digitales especializadas y, sobre todo, se toma todo tipo de decisiones.

Para alcanzar dichos beneficios, las empresas, instituciones y organizaciones han llevado la difusión de la TIC a una esfera en la cual se generen nuevos conocimientos, invenciones e innovaciones, a partir del perfeccionamiento del uso de la tecnología. Ese tipo de arreglo económico ha sido denominado, en este trabajo, "economía digital basada en el conocimiento".

Este concepto se crea para vincular la difusión de la tecnología con aspectos económicos, se define como: "el conjunto de acciones digitales que permiten generar nuevas oportunidades económicas a los usuarios de la Tic con la aplicación de sus conocimientos y habilidades sobre el dominio de la tecnología y la generación de nuevos desarrollos tecnológicos internos" (Lira, 2005). Algunas oportunidades económicas son:

\section{DeSarrollo}


1. Disminución de costos de producción y operación

2. Penetración en mercados

3. Eficiencia en procesos

4. Renta tecnológica

La idea principal es que para que la TIC produzca valor agregado y oportunidades económicas. Los conocimientos, capacidades y habilidades de los usuarios deben ser aplicados al dominio de la tecnología, en actividades y funciones cotidianas, así como en la creación de nuevos desarrollos tecnológicos.

Para lograrlo, se requiere del aprendizaje tecnológico de los usos y aplicaciones de la tecnología en procesos laborales, pues mediante éste, los usuarios tienen mayor dominio de la tecnología, lo cual posibilita la creación de nuevos desarrollos tecnológicos. ${ }^{39}$

No obstante, como lo demuestran aportaciones teóricas acerca de la gestión del conocimiento y cambio tecnológico (Villavicencio, 1989), la tecnología no es fácilmente transferible y utilizable; es específica a un cierto conjunto de aplicaciones o funciones organizacionales y su acumulación se manifiesta por medio de trayectorias que revelan una lógica de utilización y una especificidad de saberes.

En las organizaciones, el aprendizaje tecnológico puede desarrollarse por medio de procesos individuales y colectivos; ${ }^{40}$ en éstos, el conocimiento transita de un estado tácito a otro colectivo mediante un proceso en el cual el conocimiento tácito se socializa (divulga) y los conocimientos socializados (existentes en la organización) se interiorizan para generar un nuevo conocimiento tácito para el individuo y convertir estas acciones en un proceso cíclico de generación de nuevos conocimientos (Krogh, Ichijo, y Nonaka, 2000).

En la utilización de la TIC, estos procesos pueden manifestarse por medio de la exploración de las acciones de digitalización de la información, comunicaciones y mecanismos de coordinación desarrollados, en los cuales el aprendizaje puede tomar diversas formas básicas de implantación, tales como: learning by doing (aprender haciendo), learning by working (aprender trabajando), learning by trail and mistake (aprender por ensayo y error), learning by train (aprender por capacitación) y la reingeniería de reversa, entre otros. ${ }^{41}$

39 La transferencia tecnológica implica la asimilación y adaptación de la tecnología hacia los modos y formas organizacionales. Éste es un proceso continuo de interrelaciones e interacciones que hacen posible la transmisión y adquisición de saberes en cuanto a la aplicación operativa de una tecnología específica, en el cual el reto organizacional, por parte de los trabajadores, es alcanzar el dominio de la tecnología desde los procesos productivos establecidos (Villavicencio, op. cit.).

40 Los procesos organizacionales de aprendizaje tecnológico se pueden desarrollar en los niveles individual y colectivo mediante los esfuerzos internos de los integrantes de la organización o por la incorporación de nuevos (Dutrenit, 2000).

41 El cambio tecnológico, un panorama selectivo, p. 24.

\section{DeSarrollo}


En esta línea del pensamiento, el aprendizaje, que produce nuevos conocimientos ${ }^{42}$ para las organizaciones, y la aplicación de éstos en los ámbitos de competencia, puede brindar un valor económico por medio de la generación de innovaciones en producto, servicios y procesos.

Dodgson (1991) menciona que el objetivo organizacional de la gestión del conocimiento es organizar, complementar y crear conocimientos alrededor de las rutinas y de la cultura organizacional, con el fin de adaptar y desarrollar la eficiencia y competencia económica de la organización.

Este enfoque conceptual expone el dominio de la tecnología y la generación de nuevos desarrollos tecnológicos como los elementos centrales que permiten el perfeccionamiento del uso de la TIC en empresas, instituciones y organizaciones y, con ello, el alcance de beneficios económicos y operativos.

Estos resultados son posibles a medida que los usuarios de la tecnología conforman una base de conocimientos, habilidades y capacidades tecnológicas en sus acciones laborales. Este perfeccionamiento es resultado del aprendizaje tecnológico. ${ }^{43}$

\section{Comentarios finales}

Este artículo exhibió el estudio de la TIC desde un modelo de exploración que cubrió dos elementos de análisis: la difusión y el perfeccionamiento. Más allá de presentar una discusión teórica o metodológica, se analizó una estructura informativa y conceptual, mediante la compilación de argumentos teóricos y contextuales que siguieron dos ideas.

La primera fue que la exploración de la difusión de la tecnología permite el estudio del crecimiento de los usuarios de Tic y de las prácticas digitales y, con ello, de la misma sociedad de la información. Se vincularon de forma lineal los elementos: acceso —difusión—y sociedad de la información, en la cual el acceso a la tecnología permite mayor difusión en los países, la cual revela el grado de construcción de dicha sociedad. Esta idea se expone en el conjunto de mediciones e indicadores cuantitativos acerca de la sociedad de la infor-

42 En el presente estudio, el conocimiento es entendido como una certeza justificada tanto explícita como tácita (Krogh, Ichijo, y Nonaka, op. cit.) y su gestión como la manera de propiciar o facilitar el conocimiento, por medio de la búsqueda de nuevos conocimientos, utilizar, adaptar y cambiar los existentes y utilizar los actuales (Dutrenit, op. cit.). En ese sentido, se observa que las organizaciones, en su intento por integrar tecnologías emergentes en sus operaciones económicas, realizan esfuerzos para generar conocimientos que les permitan asimilar y adaptar esa tecnología y, en esa línea, intentan compartir conocimiento tácito, crear conceptos, justificarlos, elaborar prototipos e inter nivelar el conocimiento (Krogh, Ichijo y Nonaka, ibid.).

43 El aprendizaje tecnológico y la gestión del conocimiento sobre el uso de la tecnología son un meca nismo para generar capacidades tecnológicas en las organizaciones porque permiten mayor dominio de la tecnología en los procesos productivos y operaciones administrativas, lo cual deriva en una mayor eficiencia económica y competitividad de mercado en caso de ser los primeros en implantarlo (Westphal, Kim y Dahlman, 1985; Bell y Pavitt, 1995; Kim, 1997; Leonard, 1992 y 1995).

\section{DeSarrollo}


mación y también en las políticas digitales de diferentes países como Estados Unidos, Singapur, España y Chile.

La segunda idea desarrollada fue que el perfeccionamiento del uso de la TIC — por medio del dominio de la tecnología y la generación de nuevos desarrollos tecnológicos en sistemas de información, comunicaciones y mecanismos de coordinación- es el elemento central para la creación de oportunidades económicas y operativas en empresas, instituciones y organizaciones.

Se destacó que ambos aspectos son producto de mecanismos de aprendizaje tecnológico en los cuales la gestión del conocimiento, relativo a la aplicación de la tecnología en actividades especializadas, cobra primordial importancia. Con ello, se hace explícita la vinculación entre la gestión del conocimiento, mecanismos de aprendizaje, invenciones e innovaciones con el concepto economía digital basada en el conocimiento y con la generación de oportunidades y beneficios económicos y operativos con el uso de la Tic.

En ese sentido, el conjunto de aportaciones conceptuales expuso que la inserción de una organización, institución o empresa en la economía digital basada en el conocimiento requiere que los integrantes, servidores públicos o empleados usen la TIC en sus actividades (la difundan) y, con ella, generen un valor económico mediante acciones de digitalización, integrando en éstas el conjunto de información, conocimientos y habilidades organizacionales (capacidades tecnológicas).

La exploración contextual de la difusión y perfeccionamiento de la TIC en México mostró claramente que su absorción es aún incipiente y existen brechas de conectividad y digitales importantes entre las regiones, las cuales impide una difusión integral con la misma intensidad.

No obstante, es necesario denotar, conforme al contexto y literatura expuestos, que en México el reto de la política digital para los próximos años será:

1. Consolidar las políticas y estrategias de acceso a la tecnología en el conjunto de las regiones del país.

2. Propiciar un creciente uso de la TIC en las actividades de la comunidad, empresas e instituciones, con el apoyo de fuentes de financiamiento y marcos normativos que permitan la efectiva implantación de proyectos en comercio y administración electrónicas, educación a distancia y en la construcción de sistemas de información y comunicaciones digitales en temas primordiales para el crecimiento y desarrollo de México como son: campo, desarrollo industrial (manufacturas), comercio exterior, seguridad social, democracia, transparencia, educación y ciencia, y la tecnología.

3. El diseño de políticas y estrategias para propiciar la construcción de una economía digital basada en el conocimiento, como lo es el caso brasileño, más aun cuando se observa que la creación de capital humano en TIC es una de las áreas académicas prin-

\section{Desararrollo}




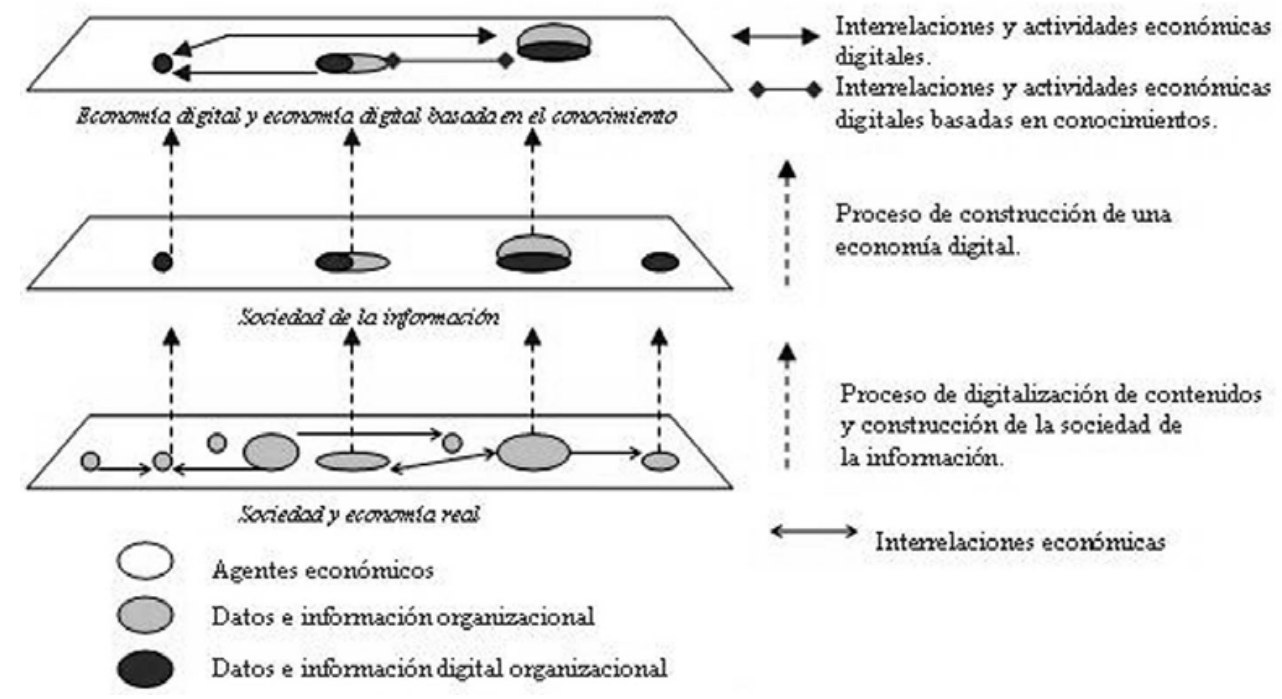

Gráfica 3. Economía de las tecnologías digitales

Fuente: elaborado con base en diversas aportaciones.

cipales de formación en los niveles técnico, superior y posgrado, así como en la investigación y desarrollo del país.

Desde esta perspectiva, se observa que el contexto sociodemográfico de las regiones es el elemento fundamental de la difusión de la tecnología y de la construcción de la sociedad de la información, en la cual los aspectos económicos, cognitivos, normativos y culturales delimitan y definen el tipo de dicha sociedad. Con ello, la política digital puede ser replanteada de un diseño macro a diseños meso (regional), que posiblemente permitirían una difusión más eficiente y efectiva de la TIC.

Finalmente, se observa que la difusión de la tecnología en empresas, instituciones y organizaciones debe trascender al logro de beneficios económicos y operativos mediante el dominio de la técnica y de la generación de nuevos desarrollos tecnológicos que, de ser innovaciones, generan valor agregado por medio de la renta tecnológica y la competitividad. Con ello, el conjunto de agentes que alcancen estos resultados estarán transitando a una economía digital basada en el conocimiento, cuyo centro sea el aprendizaje tecnológico y la gestión del conocimiento. 


\section{Bibliografía}

Andreu, R.; J. Ricart y J. Valor, "Information Systems Planning at the Corporate Level", en C. Ciborra y T. Jelassi, Strategic Information Systems, Londres, John Wisley \& Sons, 1994.

Bell, M. y K. Pavitt, "The Development of Technological Capabilities”, en I.U. Haque Trade, Technology and International Competitiveness, Washington, World Bank, 1995.

Benghozi, P. y C. Licoppe, "Technological National Learning in France: From Minitel to Internet", en B. Kogut, The Global Internet Economy, Cambridge, The мIт Press, 2003.

Burch, J. y G. Grudnitski, Diseño de sistemas de información, México, Limusa, 1989.

Casper, E. (2003) “The German internet economy and the Silicon Valley Model: Convergence, Divergence,or something else?", en B. Kogut, The Global Internet Economy, Cambridge, The MIT Press, 2003.

Castells, M., "La era de la información" vol. I, La sociedad red, 1996.

CEPAL-ECLAC, "América Latina y el Caribe en la transición hacia la sociedad del conocimiento", Santiago de Chile, junio de 2000.

CEPAL, "Los caminos hacia una sociedad de la información en América Latina y el Caribe", Santiago de Chile, julio de 2003.

Cecchini, S., "Tecnologías de información y comunicación (TIC) y equidad en América Latina", Santiago de Chile, ECLAC-CEPAL, 2004.

Ciborra, C., "The Grassroots of IT and Strategy", en C. Ciborra, y T. Jelassi, Strategic Information Systems, Londres, John Wisley \& Sons, 1994.

Cukor, P. y L. MacKnigth, "Knowledge Networks, the Internet and Development", ponencia, 28th Annual TPRC, 2000.

Chang, S., "The Internet Economy of Korea", en B. Kogut, The Global Internet Economy, Cambridge, The MIT Press, 2003.

Dodgson, M., "The Management of Technological Collaboration", en Engineering Management Journal, vol. 1, núm. 4, 1991, pp. 187-192.

Dodgson, M.; D. Gann y A. Salter, "The Intensification of Innovation", en International Journal of Innovation Management, vol. 6, núm. 1, marzo de 2002.

Dutrenit, G., "Learning and Knowledge Management in the Firm: From Knowledge Accumulation to Strategic Capabilities", Cheltenham, Northhampton, Edward Elgar, 2000.

Earl, M., "Knowledge as Strategy: Reflections on Skandia International and Shorko Films"en C.
Ciborra y T. Jelassi, Strategic Information Systems, Londres, John Wisley \& Sons, 1994.

Fine, H. y D. Raff, "Internet-Driven Innovation and Economic Performance in the American Automobile Industry", ponencia, The E-Business Transformation: Sector Developments and Policy Implications, Washington, 26-27 de septiembre de 2000.

Glimstedt, H. y U. Zander, "Sweden's Wíreless Wonders: The Diverse Roots and Selective Adaptations of the Swedish Internet Economy", en B. Kogut, The Global Internet Economy, Cambridge, The MIT Press, 2003

Hilbert, M. y J. Katz, "Building an Information Society: A Latin American and Caribbean Perspective", Santiago de Chile, CEPAL-EClaC, 2003.

Hilber, M.; S. Bustos y J. Ferraz, "Estrategias nacionales para la sociedad de la información en América Latina y el Caribe", Santiago de Chile, CEPAL-ECLAC, 2003.

INFOTEC, "Usabilidad: Un concepto que debería considerarse en todos los sitios de internet", en Puntogob, núm. 5, agosto/septiembre de 2002.

Kenney, M., "The Grown and Development of the Internet in the Unites States", en B. Kogut, The Global Internet Economy, Cambridge, The MIT Press, 2003.

Kim, L. (1997) "From Imitation to Innovation. The Dynamics of Korea's Technological Learning", Boston, Harvard Business School Press.

Kogut, B., "The Internet has Borders", en B. Kogut, The Global Internet Economy, Cambridge, The MIT Press, 2003a.

_, "From Pockets of Experimentation to Institutional Change", en B. Kogut, The Global Internet Economy, Cambridge, The MIT Press, 2003b.

Krogh, G.; Ichijo, K. y Nonaka, I., Enabling knowledge creation, Oxford University Press, 2000.

Leonard, B., "Core Capabilities and Core Rigidities: a Paradox in Managing New Product Development", en Strategic Management Journal, núm. 13, 1992, pp. 111-125.

, "Wellsprings of Knowledge", Boston, Harvard Business School Press, 1995.

Linares, J., "Principios de una ética para el mundo tecnológico", México, UNAM, 2004.

Lira, R.A., "Evolución del uso de internet en la Administración Pública: El caso de la subdirección de Estadística Informática-INEGI", México, UAM, 2005.

\section{Desars rollo}


Murdick, R. y J. Munson, "Sistemas de información administrativa", México, Prentice-Hall Hispanoamericana, 1988

Nonaka, I., "Toward Middle Up-down Management: Accelerating Information Creation", en Sloan Management Review, 1988.

Perez, C., "Las nuevas tecnologías: una visión de conjunto", en Carlos Ominami (editor), La tercera revolución industrial: impactos internacionales del actual viraje tecnológico, Buenos Aires, RIAL, Grupo Editor Latinoamericano, 1986, pp. 43-90.

Rieck, R. y K. Dickson, "A Model of Technology Strategy", en Technology Analysis and Startegic Manegement, vol. 5, núm. 4, 1993, pp. 397-412.

Sako, M. (2003) "Between Bit Valley and Silicon Valley: Hybrid Forms of Business Governance in the Japanese Internet Economy", en B. Kogut, The Global Internet Economy, Cambridge, The мIT Press, 2003.
Senn, J., "Análisis y diseño de sistemas de información”, México, Mc-GrawHill, 1992.

Villavicencio, D., "La transferencia de tecnología: un problema de aprendizaje colectivo", en Argumentos, núm. 10-11, diciembre de 1989, pp. 7-18.

Volkow, N., "La brecha digital un concepto social de cuatro dimensiones", en Boletín de Política Informática, núm. 6, 2003.

Westphal, L.; L. Kim y C. Dahlman, "Reflections of the Republic of Korea's Acquisition of Technological Capabilities", en N. Rosenberg y C. Frischtak, International Technology, Nueva York, Praeger, 1985.

Zaheer, S. y R. Rajan, "Creativity Under Constraint: Technological Impriting and the Migration of Indian Business to the New Economy", en B. Kogut, The Global Internet Economy, Cambridge, The MIT Press, 2003. 